$03,18,11$

\title{
Моделирование фазовых переходов графитов в алмазоподобные фазы
}

\author{
(C) Е.А. Беленков, В.А. Грешняков \\ Челябинский государственный университет, \\ Челябинск, Россия \\ E-mail: belenkov@csu.ru
}

(Поступила в Редакцию 27 декабря 2017 г.)

\begin{abstract}
Методом теории функционала плотности исследованы структурные преобразования между графитами и алмазоподобными фазами. Расчеты были выполнены в двух приближениях - приближении локальной плотности и обобщенном градиентном приближении. Установлено, что фазовые переходы гексагональных графеновых слоев в кубический алмаз и алмазоподобные фазы должны происходить при одноосных сжатиях 57-71 GРa, тогда как некоторые алмазоподобные фазы могут быть получены из тетрагональных графеновых слоев при значительно меньших давлениях $\sim 32-52 \mathrm{GPa}$. Рассчитаны рентгенограммы фазового перехода графита $I 4_{1} / a m d$ в тетрагональную фазу LA10, происходящего при минимальном давлении, которые могут быть использованы для экспериментальной идентификации этих соединений.
\end{abstract}

DOI: 10.21883/FTT.2018.07.46111.363

\section{1. Введение}

Углеродные материалы с алмазоподобной структурой привлекают большой интерес исследователей изза превосходных физико-технических характеристик [1]. К настоящему времени синтезировано только несколько фаз [2] и теоретически предсказаны более шести десятков таких соединений [2-7]. Поэтому необходимо найти способы экспериментального получения этих фаз. Одним из возможных способов синтеза является воздействие высокими давлениями на графитоподобные материалы $[2,8,9]$. В качестве исходных углеродных материалов для синтеза могут быть использованы конденсаты таких наноструктур-предшественников, как графеновые слои $[8,10]$, углеродные нанотрубки $[11,12]$ и фуллерены $[13,14]$. Экспериментально установлено, что наиболее перспективным предшественником для синтеза алмазоподобных фаз является графит, так как он является самым устойчивым среди прочих материаловпредшественников и наиболее распространенным в природе. Несмотря на очевидные преимущества использования графита для синтеза алмаза и алмазоподобных фаз, способы получения этих фаз при низких температурах $(T<300 \mathrm{~K})$ недостаточно изучены. Также еще не проведены исследования, касающиеся возможности использования других полиморфов графена, на основе которых можно сформировать новые алмазоподобные соединения. Поэтому в данной работе выполнено моделирование процесса фазовых превращений различных структурных модификаций графитов, состоящих из гексагональных $\left(L_{6}\right)$ или тетрагональных $\left(L_{4-8}\right)$ графеновых слоев, в кубический алмаз и алмазоподобные фазы при сильных одноосных сжатиях и низких температурах.

\section{2. Методическая часть}

Расчеты структур и энергетических характеристик углеродных соединений были выполнены в программном пакете Quantum ESPRESSO [15] при использовании
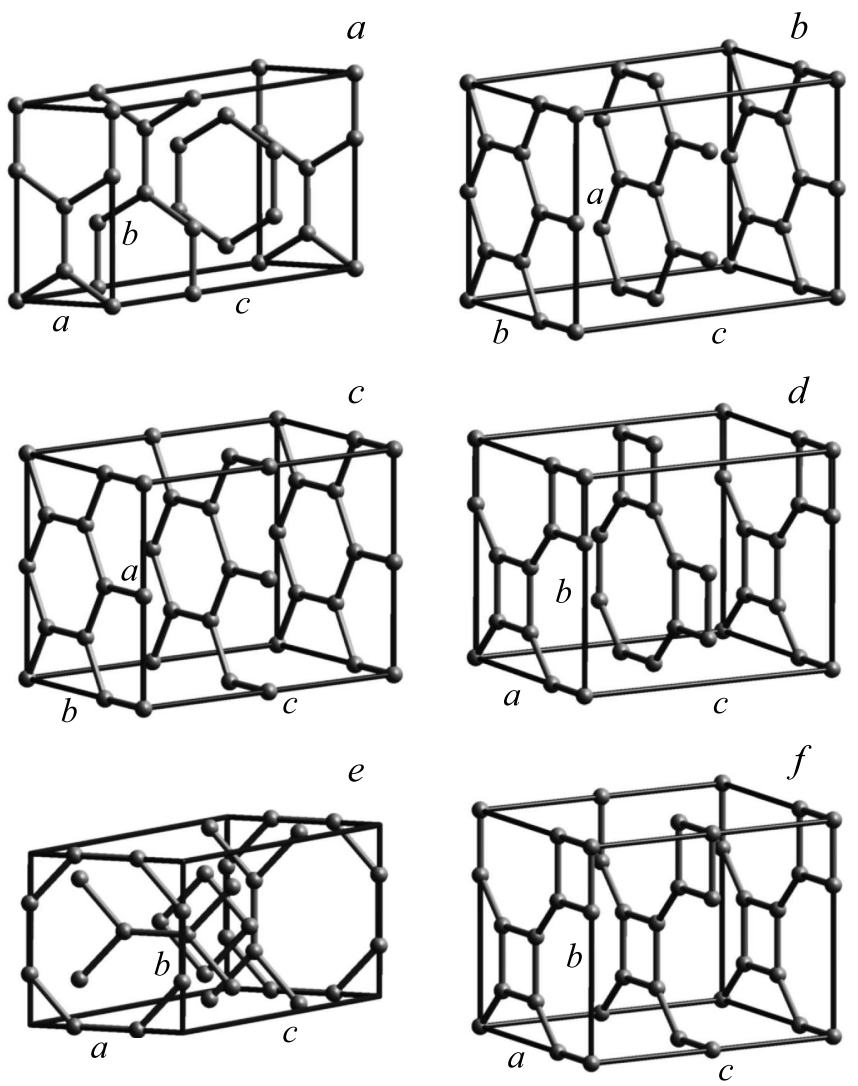

Рис. 1. Элементарные ячейки графита $L_{6} A B C(a), A B(b)$ и $A A(c)$, а также тетрагонального графита $L_{4-8} A B(d)$, $A B C D(e)$ и $A A(f)$. 

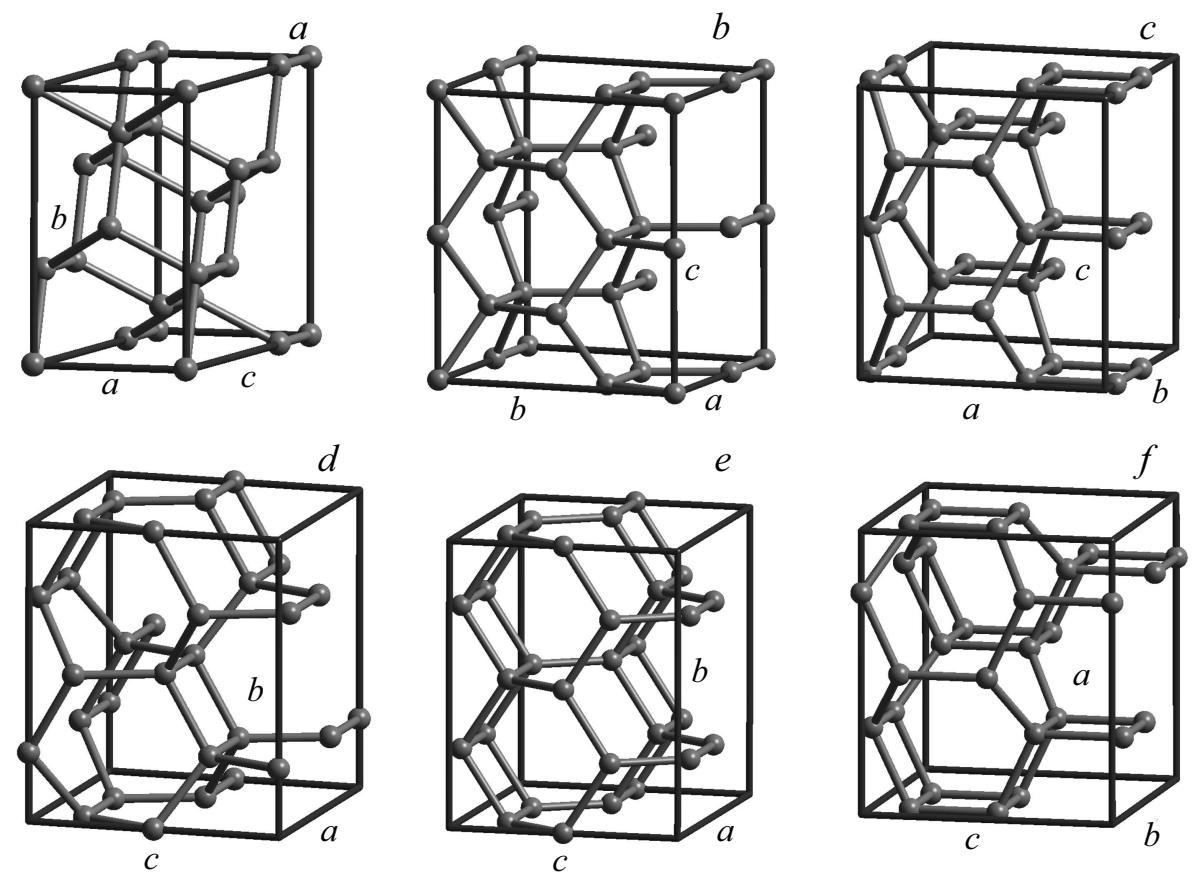

$g$
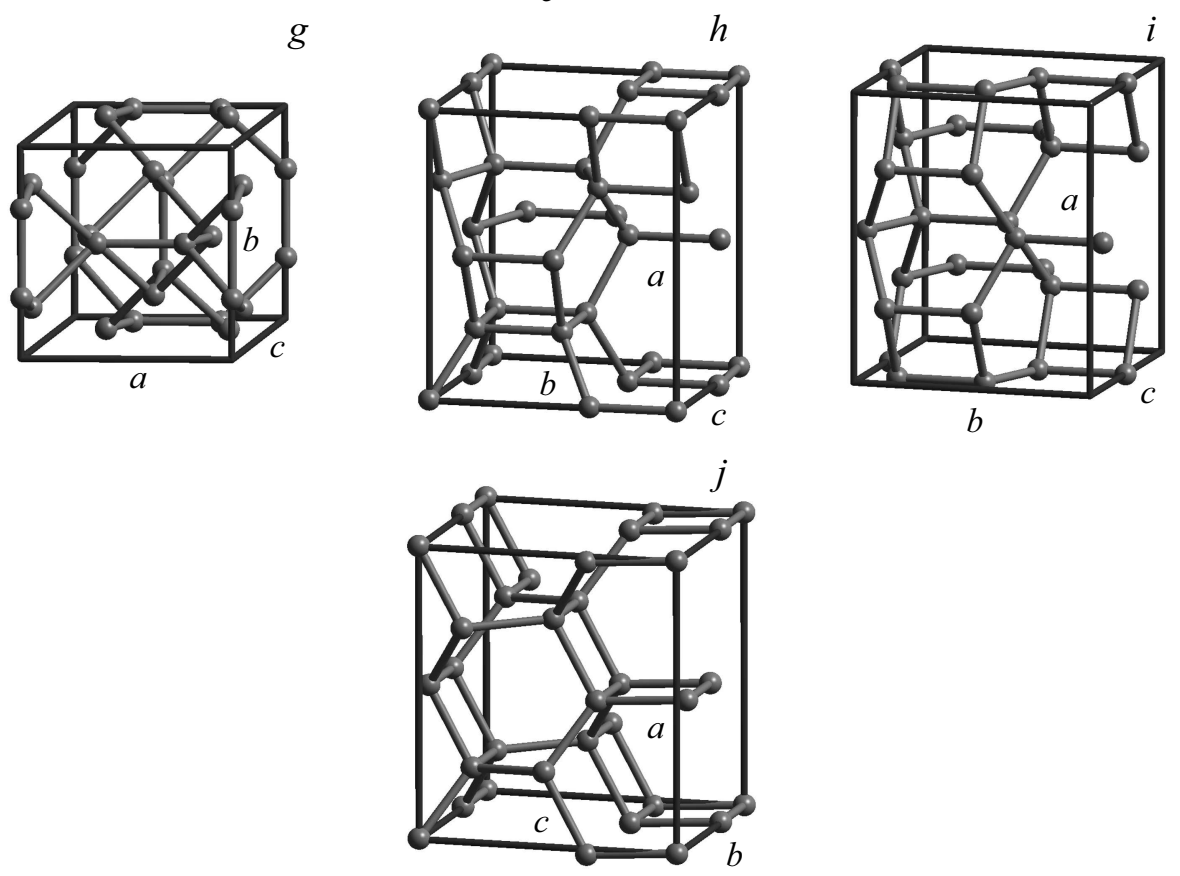

Рис. 2. Элементарные ячейки алмазоподобных фаз: кубический алмаз (a), LA2 (b), LA3 (c), LA5 (d), LA6 (e), LA7 (f), LA10 (g), $P 2_{1} / m(h)$, Рmma $(i)$ и $C 2 / m(j)$.

метода теории функционала плотности (DFT). Для расчета энергии обменного взаимодействия и электронной корреляции были использованы приближение локальной плотности (LDA) и обобщенное градиентное приближение (GGA) в формулировках Педью-Зангера (PZ) [16] Педью-Берка-Ернзерхофа (PBE) [17], соответственно. Влияние ионных остовов учитывалось через сохраняющие норму псевдопотенциалы. В расчетах использовались сетки $12 \times 12 \times 12$ из $k$-точек, определенные по методу Монхорста-Пака [18]. Разложение волновых функций производилось по усеченному базисному набору плоских волн. Размерность набора базисных функций была ограничена значением отсечки кинетической энергии $\left(E_{\text {cutoff }}\right)$, равным $700 \mathrm{eV}$.

Для модельного исследования структурных переходов кристаллов различных разновидностей графита в алмазоподобные фазы были рассмотрены элементарные ячейки, содержавшие одинаковое число углеродных атомов и имеющие близкие структурные мотивы. Расчет прямых и обратных фазовых переходов заключался в постепенном 
Таблица 1. Параметры элементарных ячеек, равновесные объемы и полные энергии углеродных $s p^{2}$ и $s p^{3}$ фаз, рассчитанные методами DFT-LDA и DFT-GGA (сингонии: T - тетрагональная; P — ромбическая; М — моноклинная)

\begin{tabular}{|c|c|c|c|c|c|c|c|c|}
\hline Фаза & Сингония & $a, \AA$ & $b, \AA$ & $c, \AA$ & $\beta,{ }^{\circ}$ & $Z$ & $V_{0}, \AA^{3} /$ atom & $E_{\text {total }}, \mathrm{eV} /$ atom \\
\hline$L_{6} A B C$ & $\mathrm{P}$ & $\begin{array}{c}2.465 \\
(2.488)\end{array}$ & $\begin{array}{c}4.270 \\
(4.309)\end{array}$ & $\begin{array}{c}9.583 \\
(11.159)\end{array}$ & $\begin{array}{c}90.00 \\
(90.00)\end{array}$ & 12 & $\begin{array}{c}8.403 \\
(9.969)\end{array}$ & $\begin{array}{l}-157.783 \\
(-157.341)\end{array}$ \\
\hline$L_{6} A B$ & $\mathrm{P}$ & $\begin{array}{c}4.265 \\
(4.308)\end{array}$ & $\begin{array}{c}4.931 \\
(4.977)\end{array}$ & $\begin{array}{c}6.428 \\
(7.361)\end{array}$ & $\begin{array}{c}90.00 \\
(90.00)\end{array}$ & 16 & $\begin{array}{l}8.450 \\
(9.864)\end{array}$ & $\begin{array}{c}-157.780 \\
(-157.339)\end{array}$ \\
\hline$L_{6} A A$ & $\mathrm{P}$ & $\begin{array}{c}4.927 \\
(4.975)\end{array}$ & $\begin{array}{c}4.265 \\
(4.308)\end{array}$ & $\begin{array}{c}6.745 \\
(7.708)\end{array}$ & $\begin{array}{c}90.00 \\
(90.00)\end{array}$ & 16 & $\begin{array}{c}8.859 \\
(10.325)\end{array}$ & $\begin{array}{c}-157.764 \\
(-157.336)\end{array}$ \\
\hline$L_{4-8} A B$ & $\mathrm{~T}$ & $\begin{array}{c}4.867 \\
(4.905)\end{array}$ & $\begin{array}{c}4.867 \\
(4.905)\end{array}$ & $\begin{array}{c}6.163 \\
(7.245)\end{array}$ & $\begin{array}{c}90.00 \\
(90.00)\end{array}$ & 16 & $\begin{array}{c}9.124 \\
(10.895)\end{array}$ & $\begin{array}{c}-157.181 \\
(-156.784)\end{array}$ \\
\hline$L_{4-8} A B C D$ & $\mathrm{~T}$ & $\begin{array}{c}3.442 \\
(3.469)\end{array}$ & $\begin{array}{c}3.442 \\
(3.469)\end{array}$ & $\begin{array}{c}12.180 \\
(13.972)\end{array}$ & $\begin{array}{c}90.00 \\
(90.00)\end{array}$ & 16 & $\begin{array}{c}9.018 \\
(10.507)\end{array}$ & $\begin{array}{c}-157.191 \\
(-157.785)\end{array}$ \\
\hline$L_{4-8} A A$ & $\mathrm{~T}$ & $\begin{array}{c}4.867 \\
(4.905)\end{array}$ & $\begin{array}{c}4.867 \\
(4.905)\end{array}$ & $\begin{array}{c}6.163 \\
(7.245)\end{array}$ & $\begin{array}{c}90.00 \\
(90.00)\end{array}$ & 16 & $\begin{array}{c}9.124 \\
(10.895)\end{array}$ & $\begin{array}{c}-157.180 \\
(-156.781)\end{array}$ \\
\hline $\begin{array}{c}3 C \text { алмаз } \\
(L A 1)\end{array}$ & $\mathrm{P}$ & $\begin{array}{l}2.513 \\
(2.545)\end{array}$ & $\begin{array}{c}4.352 \\
(4.404)\end{array}$ & $\begin{array}{c}6.155 \\
(6.229)\end{array}$ & $\begin{array}{c}90.00 \\
(90.00)\end{array}$ & 12 & $\begin{array}{c}5.609 \\
(5.820)\end{array}$ & $\begin{array}{c}-157.880 \\
(-157.278)\end{array}$ \\
\hline $\begin{array}{c}2 H \text { алмаз } \\
(L A 2)\end{array}$ & $\mathrm{P}$ & $\begin{array}{c}4.155 \\
(4.203)\end{array}$ & $\begin{array}{c}4.329 \\
(4.377)\end{array}$ & $\begin{array}{c}4.998 \\
(5.060)\end{array}$ & $\begin{array}{c}90.00 \\
(90.00)\end{array}$ & 16 & $\begin{array}{c}5.619 \\
(5.817)\end{array}$ & $\begin{array}{c}-157.850 \\
(-157.249)\end{array}$ \\
\hline$L A 3$ & $\mathrm{P}$ & $\begin{array}{c}4.353 \\
(4.401)\end{array}$ & $\begin{array}{c}4.353 \\
(4.401)\end{array}$ & $\begin{array}{c}5.002 \\
(5.055)\end{array}$ & $\begin{array}{c}90.00 \\
(90.00)\end{array}$ & 16 & $\begin{array}{c}5.924 \\
(6.120)\end{array}$ & $\begin{array}{c}-157.636 \\
(-157.065)\end{array}$ \\
\hline$L A 5$ & $\mathrm{P}$ & $\begin{array}{c}4.337 \\
(4.386)\end{array}$ & $\begin{array}{c}5.025 \\
(5.080)\end{array}$ & $\begin{array}{c}4.349 \\
(4.393)\end{array}$ & $\begin{array}{c}90.00 \\
(90.00)\end{array}$ & 16 & $\begin{array}{c}5.923 \\
(6.118)\end{array}$ & $\begin{array}{c}-157.624 \\
(-157.052)\end{array}$ \\
\hline$L A 6$ & $\mathrm{P}$ & $\begin{array}{c}4.900 \\
(4.948)\end{array}$ & $\begin{array}{c}5.079 \\
(5.144)\end{array}$ & $\begin{array}{c}4.176 \\
(4.225)\end{array}$ & $\begin{array}{c}90.00 \\
(90.00)\end{array}$ & 16 & $\begin{array}{c}6.496 \\
(6.721)\end{array}$ & $\begin{array}{l}-157.403 \\
(-156.864)\end{array}$ \\
\hline$L A 7$ & $\mathrm{P}$ & $\begin{array}{c}4.944 \\
(4.995)\end{array}$ & $\begin{array}{c}4.807 \\
(4.869)\end{array}$ & $\begin{array}{c}4.391 \\
(4.442)\end{array}$ & $\begin{array}{c}90.00 \\
(90.00)\end{array}$ & 16 & $\begin{array}{c}6.521 \\
(6.752)\end{array}$ & $\begin{array}{c}-157.349 \\
(-156.813)\end{array}$ \\
\hline$L A 10$ & $\mathrm{~T}$ & $\begin{array}{c}3.545 \\
(3.581)\end{array}$ & $\begin{array}{c}3.545 \\
(3.581)\end{array}$ & $\begin{array}{c}8.497 \\
(8.611)\end{array}$ & $\begin{array}{c}90.00 \\
(90.00)\end{array}$ & 16 & $\begin{array}{c}6.673 \\
(6.900)\end{array}$ & $\begin{array}{l}-157.262 \\
(-156.730)\end{array}$ \\
\hline$P 2_{1} / m$ & $\mathrm{M}$ & $\begin{array}{c}5.020 \\
(5.077)\end{array}$ & $\begin{array}{c}4.330 \\
(4.381)\end{array}$ & $\begin{array}{c}4.375 \\
(4.424)\end{array}$ & $\begin{array}{c}89.53 \\
(89.47)\end{array}$ & 16 & $\begin{array}{c}5.944 \\
(6.150)\end{array}$ & $\begin{array}{c}-157.585 \\
(-157.013)\end{array}$ \\
\hline Pmma & $\mathrm{P}$ & $\begin{array}{c}5.071 \\
(5.130)\end{array}$ & $\begin{array}{c}4.320 \\
(4.373)\end{array}$ & $\begin{array}{c}4.342 \\
(4.388)\end{array}$ & $\begin{array}{c}90.00 \\
(90.00)\end{array}$ & 16 & $\begin{array}{c}5.945 \\
(6.152)\end{array}$ & $\begin{array}{c}-157.580 \\
(-157.008)\end{array}$ \\
\hline$C 2 / m$ & M & $\begin{array}{c}5.010 \\
(5.067)\end{array}$ & $\begin{array}{c}4.478 \\
(4.533)\end{array}$ & $\begin{array}{c}4.383 \\
(4.433)\end{array}$ & $\begin{array}{c}89.31 \\
(89.24)\end{array}$ & 16 & $\begin{array}{c}6.145 \\
(6.363)\end{array}$ & $\begin{array}{c}-157.472 \\
(-156.916)\end{array}$ \\
\hline
\end{tabular}

одноосном сжатии или растяжении элементарных ячеек и нахождении их равновесной структуры. Давление $(P)$ в системе определялось из основного термодинамического тождества при температуре, близкой к абсолютному нулю. Энтальпия соединений определялась по стандартной формуле $\left(H=E_{\text {total }}+P \cdot V\right.$, где $E_{\text {total }}$ и $V-$ полная энергия и объем системы соответственно). Тепловой выход фазового перехода $\left(\Delta H_{G-D}\right)$ графитов в алмазоподобные фазы рассчитывался как разница энтальпий алмазоподобных фаз $\left(H_{\mathrm{Diam}}\right)$ и графитов $\left(H_{\mathrm{Graph}}\right)$.

Рентгенограммы поликристаллических углеродных соединений были рассчитаны в процессе сжатия и декомпрессии при использовании стандартной методики [19] для характеристического излучения $\mathrm{Cu}-K_{\alpha 1}$. Форма профилей дифракционных максимумов была описана функциями псевдо-Войгта. Средний размер кристаллитов составлял $500 \AA$.

\section{3. Результаты и обсуждения}

В качестве исходных соединений, из которых могут быть сформированы алмазоподобные фазы, были использованы графиты $L_{6}$ с основными упаковками слоев $A B C$ (пространственная группа $R \overline{3} m), A B(C m m m)$ и $A A(P 6 / \mathrm{mmm})$ (рис. $1, a-c)$ и тетрагональные гра- 
Таблица 2. Характеристики прямых и обратных фазовых превращений графитов $(G)$ в алмазоподобные фазы $(D)$ (пространственные группы и $[h k l]$-направления деформации алмазоподобных фаз, а также атомарные объемы $\left.V_{\text {trans }}\right)$, энергетические барьеры $(\Delta E)$, давления $(P)$ и изменение энтальпии $(\Delta H)$ фазовых переходов). Значения, рассчитанные методом DFT-LDA, приведены без скобок, а методом DFT-GGA — в скобках

\begin{tabular}{|c|c|c|c|c|c|c|c|c|}
\hline Графит & Фаза & Пр. группа & {$[h k l]$} & $V_{\text {trans }}, \AA^{3} /$ atom & $\Delta E_{G-D}, \mathrm{eV} /$ atom & $\Delta E_{D-G}, \mathrm{eV} /$ atom & $P_{G-D}, \mathrm{GPa}$ & $\Delta H_{G-D}, \mathrm{eV} /$ atom \\
\hline$L_{6} A B C$ & $L A 1$ & $F d \overline{3} m$ & {$[111]$} & $\begin{array}{c}6.329 \\
(6.487)\end{array}$ & $\begin{array}{c}0.216 \\
(0.316)\end{array}$ & $\begin{array}{c}0.313 \\
(0.252)\end{array}$ & $\begin{array}{c}59.0 \\
(68.0)\end{array}$ & $\begin{array}{c}-065 \\
(-0.61)\end{array}$ \\
\hline$L_{6} A B$ & \multirow{3}{*}{$L A 2$} & \multirow{3}{*}{$P 6_{3} / m m c$} & {$[100]$} & $\begin{array}{c}6.332 \\
(6.495)\end{array}$ & $\begin{array}{c}0.241 \\
(0.337)\end{array}$ & $\begin{array}{c}0.310 \\
(0.246)\end{array}$ & $\begin{array}{c}61.0 \\
(68.0)\end{array}$ & $\begin{array}{c}-0.67 \\
(-0.58)\end{array}$ \\
\hline$L_{6} A A$ & & & {$[001]$} & $\begin{array}{c}6.452 \\
(6.599)\end{array}$ & $\begin{array}{c}0.275 \\
(0.391)\end{array}$ & $\begin{array}{c}0.361 \\
(0.303)\end{array}$ & $\begin{array}{c}56.5 \\
(65.8)\end{array}$ & $\begin{array}{c}-0.65 \\
(-0.81)\end{array}$ \\
\hline$L_{6} A A$ & & & {$[110]$} & $\begin{array}{c}6.395 \\
(6.567)\end{array}$ & $\begin{array}{c}0.297 \\
(0.404)\end{array}$ & $\begin{array}{c}0.383 \\
(0.317)\end{array}$ & $\begin{array}{c}59.5 \\
(67.2)\end{array}$ & $\begin{array}{c}-0.74 \\
(-0.61)\end{array}$ \\
\hline$L_{6} A A$ & \multirow{2}{*}{$L A 3$} & \multirow{2}{*}{$I 4 / \mathrm{mmm}$} & {$[100]$} & $\begin{array}{c}6.409 \\
(6.576)\end{array}$ & $\begin{array}{c}0.292 \\
(0.400)\end{array}$ & $\begin{array}{c}0.164 \\
(0.128)\end{array}$ & $\begin{array}{c}58.8 \\
(66.8)\end{array}$ & $\begin{array}{c}-0.45 \\
(-0.34)\end{array}$ \\
\hline$L_{4-8} A A$ & & & {$[001]$} & $\begin{array}{c}7.056 \\
(7.244)\end{array}$ & $\begin{array}{c}0.207 \\
(0.295)\end{array}$ & $\begin{array}{c}0.661 \\
(0.578)\end{array}$ & $\begin{array}{c}49.8 \\
(51.9)\end{array}$ & $\begin{array}{c}-1.04 \\
(-0.97)\end{array}$ \\
\hline$L_{6} A B$ & \multirow{3}{*}{$L A 5$} & \multirow{3}{*}{ Cmca } & {$[010]$} & $\begin{array}{c}6.286 \\
(6.443)\end{array}$ & $\begin{array}{c}0.258 \\
(0.360)\end{array}$ & $\begin{array}{c}0.102 \\
(0.072)\end{array}$ & $\begin{array}{c}64.0 \\
(71.4)\end{array}$ & $\begin{array}{c}-0.29 \\
(-0.25)\end{array}$ \\
\hline$L_{6} A A$ & & & {$[100]$} & $\begin{array}{c}6.405 \\
(6.567)\end{array}$ & $\begin{array}{c}0.294 \\
(0.404)\end{array}$ & $\begin{array}{c}0.154 \\
(0.120)\end{array}$ & $\begin{array}{c}59.0 \\
(67.2)\end{array}$ & $\begin{array}{c}-0.47 \\
(-0.40)\end{array}$ \\
\hline$L_{4-8} A B$ & & & {$[001]$} & $\begin{array}{c}7.098 \\
(7.273)\end{array}$ & $\begin{array}{c}0.195 \\
(0.283)\end{array}$ & $\begin{array}{c}0.638 \\
(0.552)\end{array}$ & $\begin{array}{c}45.5 \\
(49.9)\end{array}$ & $\begin{array}{c}-0.99 \\
(-0.94)\end{array}$ \\
\hline$L_{6} A A$ & \multirow[b]{2}{*}{$L A 6$} & \multirow[b]{2}{*}{ Imma } & {$[100]$} & $<6.2$ & $>0.6$ & - & $>100$ & - \\
\hline$L_{4-8} A A$ & & & {$[010]$} & $\begin{array}{c}7.228 \\
(7.461)\end{array}$ & $\begin{array}{c}0.158 \\
(0.230)\end{array}$ & $\begin{array}{c}0.379 \\
(0.313)\end{array}$ & $\begin{array}{c}41.2 \\
(43.9)\end{array}$ & $\begin{array}{c}-0.59 \\
(-0.54)\end{array}$ \\
\hline$L_{6} A A$ & \multirow[b]{2}{*}{$L A 7$} & \multirow[b]{2}{*}{$\mathrm{Cmcm}$} & {$[010]$} & $<6.2$ & $>0.6$ & - & $>100$ & - \\
\hline$L_{4-8} A B$ & & & {$[100]$} & $\begin{array}{c}7.168 \\
(7.372)\end{array}$ & $\begin{array}{c}0.176 \\
(0.253)\end{array}$ & $\begin{array}{c}0.101 \\
(0.283)\end{array}$ & $\begin{array}{c}42.5 \\
(46.4)\end{array}$ & $\begin{array}{c}-0.52 \\
(-0.48)\end{array}$ \\
\hline$L_{4-8} A B C D$ & $L A 10$ & $I 4_{1} /$ amd & {$[100]$} & $\begin{array}{c}7.345 \\
(7.488)\end{array}$ & $\begin{array}{c}0.106 \\
(0.188)\end{array}$ & $\begin{array}{c}0.177 \\
(0.133)\end{array}$ & $\begin{array}{c}32.3 \\
(39.8)\end{array}$ & $\begin{array}{c}-0.33 \\
(-0.32)\end{array}$ \\
\hline$L_{6} A A$ & $P 2{ }_{1} m$ & $P 2_{1} / m$ & {$[010]$} & $\begin{array}{c}6.402 \\
(6.558)\end{array}$ & $\begin{array}{c}0.164 \\
(0.408)\end{array}$ & $\begin{array}{c}0.115 \\
(0.084)\end{array}$ & $\begin{array}{c}59.0 \\
(67.6)\end{array}$ & $\begin{array}{c}-0.49 \\
(-0.35)\end{array}$ \\
\hline$L_{6} A A$ & Pmma & Pmma & {$[010]$} & $\begin{array}{c}6.397 \\
(6.555)\end{array}$ & $\begin{array}{c}0.296 \\
(0.409)\end{array}$ & $\begin{array}{c}0.112 \\
(0.081)\end{array}$ & $\begin{array}{c}59.4 \\
(67.7)\end{array}$ & $\begin{array}{c}-0.33 \\
(-0.36)\end{array}$ \\
\hline$L_{6} A A$ & $C 2 / m$ & $C 2 / m$ & {$[010]$} & $\begin{array}{c}6.346 \\
(6.504)\end{array}$ & $\begin{array}{c}0.186 \\
(0.431)\end{array}$ & $\begin{array}{c}0.024 \\
(0.011)\end{array}$ & $\begin{array}{c}64.3 \\
(70.0)\end{array}$ & $\begin{array}{c}-0.01 \\
(-0.17)\end{array}$ \\
\hline
\end{tabular}

фиты $L_{4-8}$ с упаковками слоев $A B C D \quad\left(I 4_{1} / a m d\right)$, $A B(I 4 / \mathrm{mmm})$ и $A A(P 4 / \mathrm{mmm})$ (рис. $1, d-f)$. Конечные соединения, структуры которых изначально были получены по методике [7,20], представлены следующим набором алмазоподобных фаз: кубический алмаз (LA1), $2 H$ политип алмаза (LA2), bct $C_{4}(L A 3), Y$-углерод (LA5), LA6, LA7, LA10, P21/m, Pmma и C2/m (рис. 2). В табл. 1 приведены параметры элементарных ячеек всех изученных фаз, рассчитанные методами DFT-LDA и DFT-GGA. Значения длин ковалентных связей в кубическом алмазе и графите, рассчитанные этими ме- тодами, отличаются от экспериментальных значений [9] на величину, не превосходящую $3.5 \%$. Разница экспериментального [9] и расчетных значений расстояния между графеновыми слоями в $3 R$ графите составляет $4.7 \%$ для метода DFT-LDA и 10.9\% для DFT-GGA.

Теоретический анализ способов получения алмазоподобных соединений показал, что из графита $L_{6} A B C$ возможно получение только кубического алмаза, а из гипотетического графита $L_{4-8} A B C D$ - фазы $L A 10$. Из графита $L 6 A A$ могут быть сформированы все алмазоподобные фазы, кроме $L A 1$ и $L A 10$, тогда как из графита 
$L_{6} A B$ - только гексагональный алмаз и фаза $L A 5$. На основе графита $L_{4-8} A B$ могут быть получены фазы $L A 5$ и $L A 7$, а из графита $L_{4-8} A A-$ фазы $L A 3$ и $L A 6$.

Для моделирования процесса прямых и обратных фазовых переходов „графиты $\leftrightarrow$ алмазоподобные фазы“ производилось сжатие всех графитов перпендикулярно плоскостям графеновых слоев, а деформация структур алмазоподобных фаз выполнялась вдоль кристаллографических направлений с наилучшим совпадением структурных мотивов начальной и конечной фаз (табл. 2).

Графики зависимостей разностной полной энергии $\left(\Delta E_{\text {total }}\right)$ от объема $(V)$ для ромбоэдрического графита $A B C$ и кубического алмаза, характеризующие фазовые переходы, приведены на рис. 3. Для структурного преобразования $R \overline{3} m$ графита в алмаз необходимо преодолеть энергетический барьер $\Delta E_{G-D}=0.216(0.316) \mathrm{eV} /$ atom (далее по тексту в скобках будут указаны величины, полученные с помощью метода DFT-GGA). Данное структурное преобразование должно происходить в точке пересечения функций зависимости $\Delta E_{\text {total }}$ от объема для различных фаз, т.е. при величинах $V$ от 6.329 до $6.487 \AA^{3}$ /atom, которые соответствуют давлениям от 59 до $68 \mathrm{GPa}$ (табл. 2). В результате структурного перехода ромбоэдрического графита в алмаз происходит скачкообразное уменьшение величины плотности на 14\%. Рассчитанная величина давления прямого фазового перехода хорошо объясняет то, что макрокристаллы алмаза не формируются из графита при низких температурах $(T<300 \mathrm{~K})$ и давлениях ниже $60 \mathrm{GPa}$ [8]. Энергетический барьер для обратного фазового перехода $\left(\Delta E_{D-G}\right)$ кубического алмаза в $3 R$ графит составляет $0.252-0.313 \mathrm{eV} /$ atom. Используя полученное значение $\Delta E_{D-G}$ и величину кинетической энергии атома (для кристалла $E_{\text {kin }} \sim 3 k_{\mathrm{B}} T$ ), можно выполнить грубую оценку минимальной температуры фазового перехода $\left(T_{\text {trans }}\right)$. Расчетное значение $T_{\text {trans }} \approx 1000-1200 \mathrm{~K}$ удовлетворительно согласуется с экспериментально измеренной температурой фазового превращения алмаза в графит $\left(T_{\text {trans }} \sim 1500 \mathrm{~K}\right.$ при нормальном давлении [9]). Расчеты энтальпии углеродных фаз методом DFT-GGA показали, что структурный переход $R \overline{3} m$ графита в кубический алмаз будет сопровождаться выделением энергии, величина которой составляет от 0.61 до $0.65 \mathrm{eV} /$ atom.

Результаты расчета структурных и энергетических характеристик в процессе превращения графита $L_{6}$ с упаковкой $A B$ в алмазоподобные фазы приведены на рис. 3. Преобразование графита в $2 H$ политип алмаза и орторомбическую фазу LA5 будет происходить при давлениях от 61 до $71 \mathrm{GPa}$ (табл. 2) в результате преодоления энергетических барьеров, превышающих рассчитанное ранее $\Delta E_{G-D}$ для формирования кубического алмаза на 7-19\%. В процессе прямых фазовых переходов плотность материала будет увеличиваться на $8.7-14.2 \%$ при изменении энтальпии от -0.67 до $-0.25 \mathrm{eV} /$ atom (табл. 2). В большинстве работ, посвященных исследованиям фазовых переходов графитов в
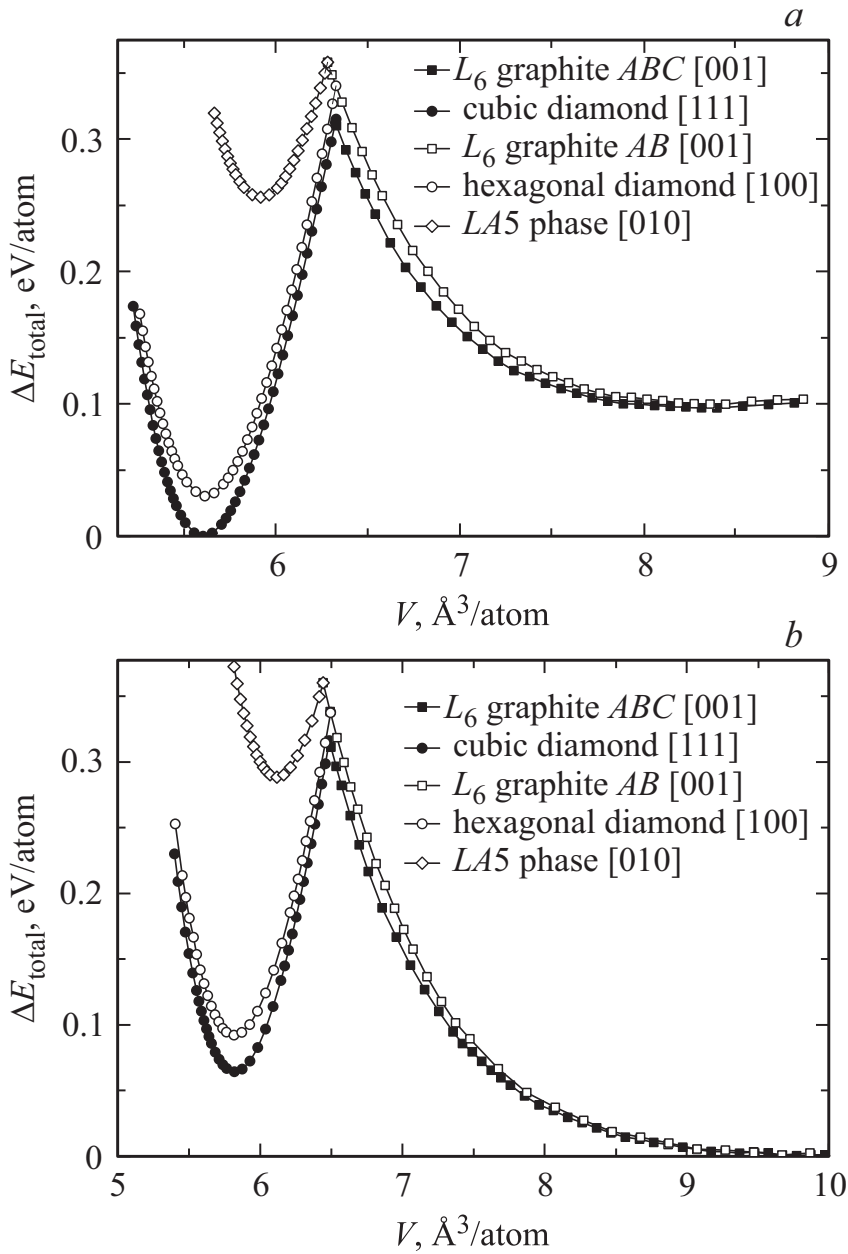

Рис. 3. Зависимости разностной полной энергии $\left(\Delta E_{\text {total }}\right)$ от объема $(V)$ для структурных превращений „графит $L_{6}$ $A B C$-кубический алмаз“ и „графит $L_{6} A B$-алмазоподобные фазы“, рассчитанные методами DFT-LDA $(a)$ и DFT-GGA $(b)$.

алмазоподобные фазы $[6,21]$, в качестве достаточного условия перехода выбрано равенство энтальпий исходной и конечной фаз. Однако изменение энтальпии, как термодинамического потенциала, при постоянном давлении представляет собой тепловой выход реакции, поэтому равенство его нулю $\left(\Delta H_{G-D}=0\right)$ не является достаточным критерием протекания фазового перехода. Стоит отметить, что в общем случае величина $\Delta H_{G-D}$ не равна нулю и указывает на экзотермический или эндотермический характер фазового перехода [22,23], что и показали выполненные в данной работе расчеты.

Рассчитанные зависимости разностной полной энергии от объема, которые характеризуют структурные преобразования между разновидностью графита $L_{6} A A$ и алмазоподобными фазами изображены на рис. 4. Минимальное давление прямого фазового перехода $\sim 57$ (66) GPa наблюдаются для $2 H$ политипа алмаза, максимальное $\sim 64(70) \mathrm{GPa}$ - для моноклинной фазы $C 2 / m$. Для инициирования структурного перехода графита $L_{6} A A$ в фазы $L A 6$ и $L A 7$ необходимо давление, 

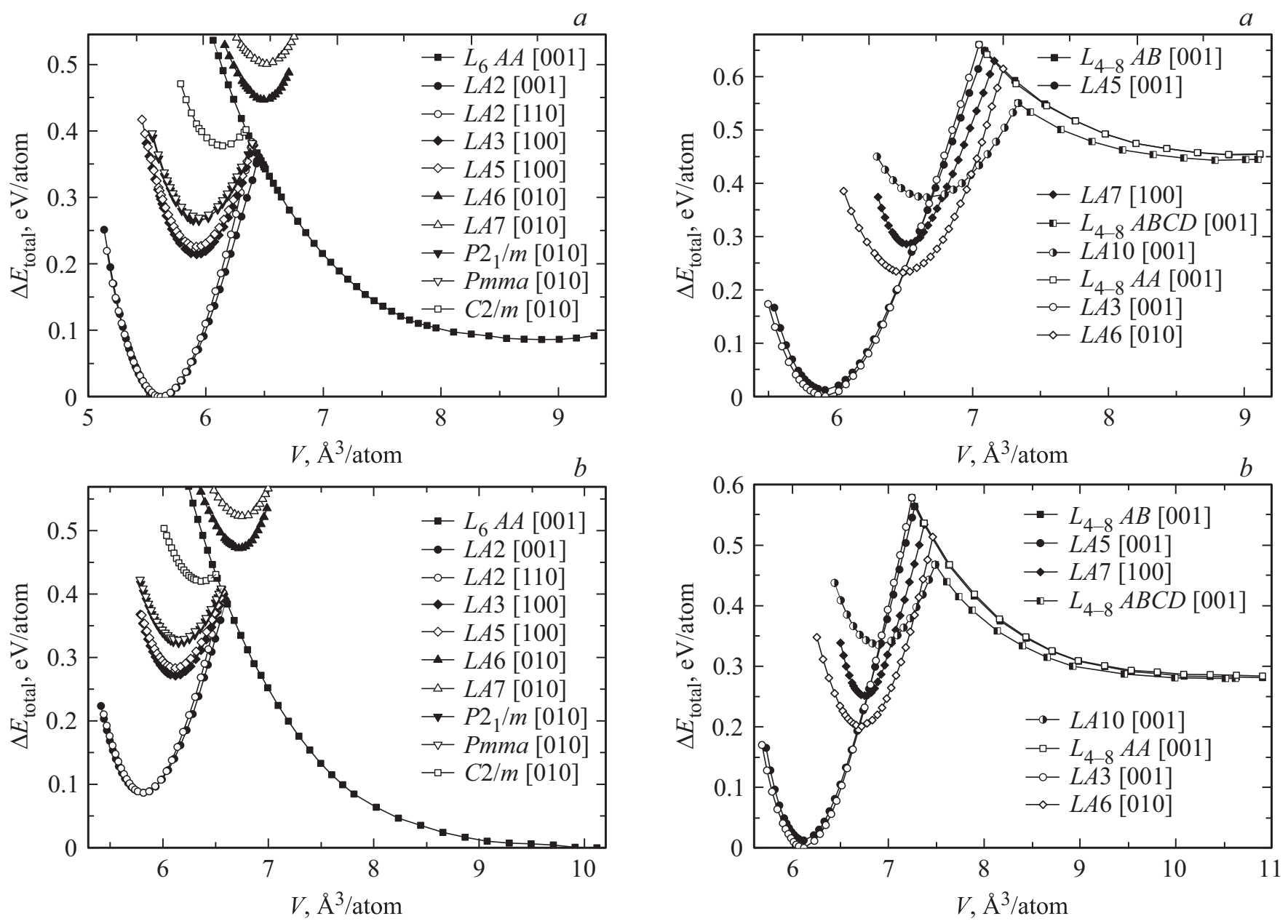

Рис. 4. Графики зависимостей разностной полной энергии $\left(\Delta E_{\text {total }}\right)$ от объема $(V)$ для фазовых переходов „графит $L_{6} A A$-алмазоподобные фазы“, рассчитанные методами DFT-LDA $(a)$ и DFT-GGA $(b)$.

превышающее 100 или $160 \mathrm{GPa}$ по результатам расчетов методами DFT-LDA и DFT-GGA соответственно. Анализ данных показал, что минимальные значения $\Delta E_{G-D}$ соответствуют фазам с минимальными значениями $E_{\text {total }}$, и, наоборот, минимальные значения $\Delta E_{\mathrm{D}-\mathrm{G}}$ имеют фазы с максимальными значениями $E_{\text {total }}$ (см. табл. 1 и 2). Все прямые фазовые переходы будут носить экзотермический характер, т. К. $\Delta H_{G-D}$ изменяется в пределах от -0.74 до $-0.01 \mathrm{eV} /$ atom.

Графики, характеризующие структурные преобразования тетрагональных графитов $L_{4-8}$ и алмазоподобных фаз $L A 3, L A 5-L A 7$ и $L A 10$, представлены на рис. 5. Прямые структурные переходы графита $L_{4-8}$ в фазы $L A 7$, $L A 6$ и $L A 10$ могут происходить при давлениях, которые на 30 (32), 30 (35) и 41 (45)\% меньше соответствующего давления, необходимого для формирования кубического алмаза (табл. 2). Значительно меньшие величины этих давлений объясняются относительно низкими энергетическими барьерами, разделяющими графиты $L_{4-8}$ и фазы $L A 6, L A 7$ и $L A 10$ (табл. 2). Формирование фаз $L A 3$ и $L A 5$ из графитов $L_{4-8} A B$ и $A A$ менее вероятно, так как

Рис. 5. Зависимости разностной полной энергии $\left(\Delta E_{\text {total }}\right)$ от объема $(V)$ для структурных превращений между графитами $L_{4-8}$ и алмазоподобными фазами, рассчитанные методами DFT-LDA $(a)$ и DFT-GGA $(b)$.

давления, необходимые для их получения, на 3-9 GPa выше соответствующих величин для фаз LA6 и LA7. Стоит отметить, что реальная величина энергетического барьера $\Delta E_{D-G}$ для фаз $L A 3$ и $L A 5$ будет, как минимум, в два раза меньше величины, указанной в табл. 2 , т.к. при обратном фазовом переходе эти фазы могут преобразоваться в $L A 6$ и $L A 7$ с последующим преобразованием в тетрагональный графит. Эта цепочка преобразований возможна при пересечении зависимостей $E_{\text {total }}=f(V)$ для различных соединений, которые можно наблюдать на рис. 5. Структурные переходы графитов $L_{4-8}$ в алмазоподобные фазы представляют собой экзотермические фазовые переходы первого рода, в результате которых будет происходить скачкообразное уменьшение объема системы на $11.4-18.7 \%$ и выделение энергии в виде тепла $\sim 0.32-1.04 \mathrm{eV} /$ atom (табл. 2).

На графиках, приведенных на рис. $3-5$, изображены зависимости от атомарного объема фаз, который является функцией давления. Зависимости изменения атомарного объема от давления для алмазоподобных фаз, получающихся из графита при минимальных давлениях, 

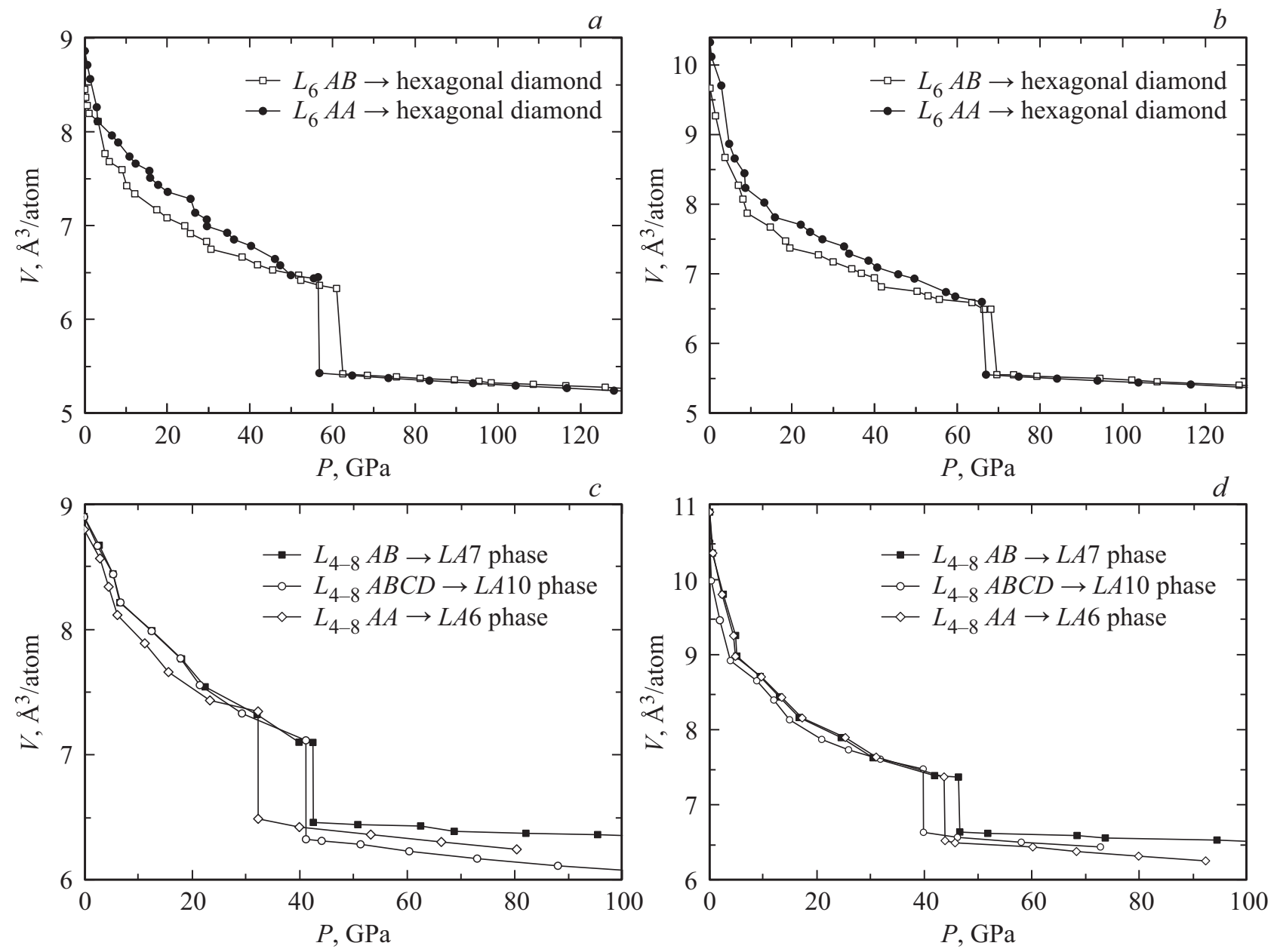

Рис. 6. Графики расчетных зависимостей атомарного объема от давления для фазовых переходов графита $L_{6}$ в гексагональный алмаз $(a, b)$ и графита $L_{4-8}$ в фазы $L A 6, L A 7$ и $L A 10(c, d)$, построенных по результатам расчетов методами DFT-LDA $(a, c)$ и DFT-GGA $(b, d)$.

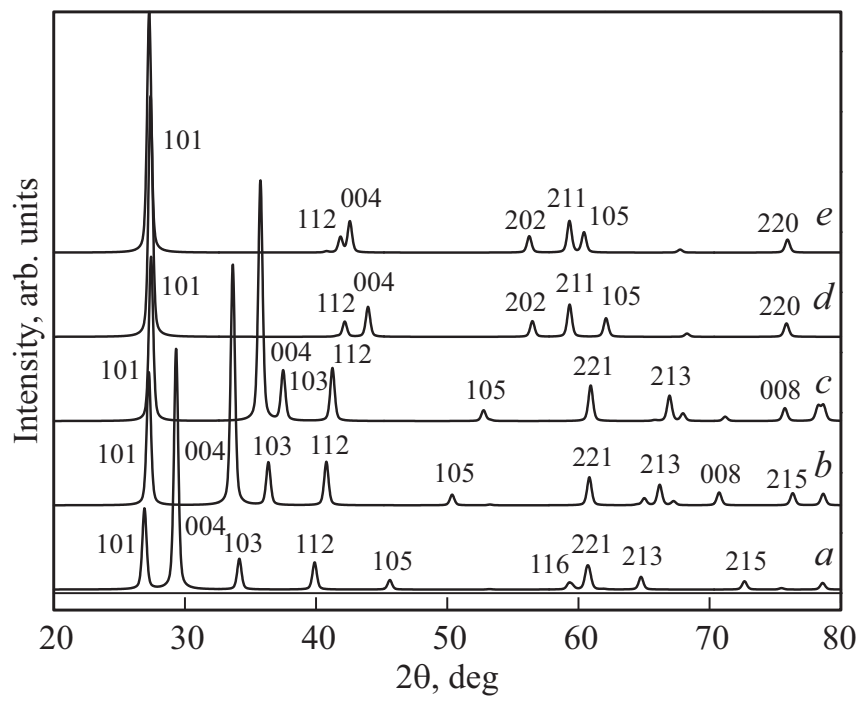

Рис. 7. Рентгенограммы углеродного материала в процессе структурного превращения при различных давлениях: $(a),(b)$ и $(c)$ - графит $L_{4-8} I 4_{1} /$ amd при 0,11 и $28 \mathrm{GPa}$ соответственно; $(d)$ и $(e)$ - тетрагональная фаза $L A 10$ при 33 и $0 \mathrm{GPa}$ соответственно. приведены на рис. 6. Давления, при которых происходят фазовые превращения, соответствуют точкам на графиках, где происходит скачкообразное изменение объема фаз. Из графитов $L_{6} A B$ и $A A$, в первую очередь, должен получаться гексагональный алмаз при давлениях 61 (68) и 57 (66) GPa соответственно (рис. 6, $a, b$ ). При сжатии графитов $L_{4-8} A B, A B C D$ и $A A$ наиболее вероятен синтез алмазоподобных фаз $L A 7, L A 10$ и LA6 при давлениях 42 (46), 32 (40) и 41 (44) GPa соответственно (рис. 6, $c, d$ ).

На заключительном этапе исследования был проведен расчет порошковых рентгенограмм материала, испытывающего фазовое превращение из тетрагонального графита с упаковкой $A B C D$ в фазу $L A 10$. Выбор именно этого фазового перехода для расчета рентгенограмм был обусловлен тем, что он должен происходить при минимальном давлении по сравнению с другими изученными фазовыми превращениями и, поэтому, может быть реализован экспериментально в первую очередь. Значения параметров элементарных ячеек и координат базиса, необходимые для расчета рентгенограмм, были вычислены с помощью метода DFT-LDA, т. к. рассчитанные с помощью него структурные характеристики 
наименьшим образом отличаются от соответствующих экспериментальных величин. При сжатии графита по оси [001] до давления $33 \mathrm{GPa}$ его наиболее интенсивный максимум 004 сильно смещается в область больших углов (с 29.3 до $36.2^{\circ}$ ), тогда как второй интенсивный максимум 101 смещается только на $0.5^{\circ}$, но при этом его относительная интенсивность увеличивается в два раза (рис. 7). При фазовом переходе происходит значительное изменение дифракционной картины, сопровождающееся исчезновением исходных максимумов низкой интенсивности и появлением множества новых максимумов (рис. 7). В процессе декомпрессии полученной фазы LA10 интенсивности и угловые позиции большей части максимумов на дифракционной картине почти не изменяются. Контролировать процесс фазового превращения можно также измеряя электрическое сопротивление материала. Как показывают расчеты, трансформация графита в фазу LA10 при $33 \mathrm{GPa}$ сопровождается скачкообразным увеличением ширины запрещенной зоны с 0 до $4.75 \mathrm{eV}$, а при декомпрессии полученной фазы $L A 10$ происходит постепенное увеличение запрещенной зоны на $3 \%$.

\section{4. Заключение}

В данной работе выполнены $a b$ initio расчеты фазовых переходов гексагональных $\left(L_{6}\right)$ и тетрагональных $\left(L_{4-8}\right)$ графеновых слоев в кубический алмаз и алмазоподобные фазы. Установлено, что из графита на основе слоев $L_{6}$ при давлениях от 57 до $71 \mathrm{GPa}$ наиболее вероятен синтез алмазоподобных фаз, имеющих минимальные значения полной энергии. Более напряженные по сравнению с кубическим алмазом алмазоподобные фазы можно получить из новых предшественников - тетрагональных графитов $L_{4-8}-$ при значительно меньших давлениях (от 32 до $52 \mathrm{GPa}$ ). Значительно меньшие величины давлений фазовых переходов тетрагонального графита $L_{4-8}$ в алмазоподобные фазы по сравнению с давлениями фазового превращения из гексагонального графена обусловлены тем, что структура графеновых слоев $L_{4-8}$ находится в более напряженном состоянии по сравнению со структурой обычного гексагонального графена. Наиболее вероятный способ синтеза изученных алмазоподобных фаз заключается в сильном одноосном сжатии различных разновидностей графита по осям, перпендикулярным графеновым слоям. Для возможности экспериментальной идентификации новой тетрагональной фазы $L A 10$, которая может быть получена при минимальном давлении (32 GPa), рассчитаны рентгенограммы фазовых переходов , $L_{4-8} \quad I 4_{1} /$ amd $\rightarrow L A 10^{\varsigma}$.

Е.А. Беленков благодарит Фонд перспективных научных исследований ЧелГУ за финансовую поддержку исследования. В.А. Грешняков благодарит РФФИ за финансовую поддержку проведенного исследования (проект № 16-33-00030 мол_а).

\section{Список литературы}

[1] E. Wilks, J. Wilks. Properties and applications of diamond. Butterworth-Heinemann Ltd., Oxford (1994). 525 p.

[2] Е.А. Беленков, В.А. Грешняков. ФТТ 58, 10, 2069 (2016).

[3] M. Takagi, T. Taketsugu, H. Kino, Y. Tateyama, K. Terakura, S. Maeda. Phys. Rev. B 95, 184110 (2017).

[4] Д.С. Лисовенко, Ю.А. Баимова, Л.Х. Рысаева, В.А. Городцов, С.В. Дмитриев. ФТТ 59, 4, 801 (2017).

[5] D.S. Lisovenko, J.A. Baimova, L.Kh. Rysaeva, V.A. Gorodtsov, A.I. Rudskoy, S.V. Dmitriev. Phys. Status Solidi B 253, 7, 1295 (2016).

[6] I.A. Baburin, D.M. Proserpio, V.A. Saleev, A.V. Shipilova. Phys. Chem. Chem. Phys. 17, 1332 (2015).

[7] Е.А. Беленков, В.А. Грешняков. ЖЭТФ 145, 1, 116 (2014).

[8] F.P. Bundy, W.A. Bassett, M.S. Weathers, R.J. Hemley, H.K. Mao, A.F. Goncharov. Carbon 34, 2, 141 (1996).

[9] H.O. Pierson. Handbook of carbon, graphite, diamond, and fullerenes: properties, processing and applications. Noyes, Park Ridge (1993). 402 p.

[10] V.D. Blank, B.A. Kulnitskiy. Int. J. Nanotechnol. 13, 8/9, 604 (2016).

[11] Z. Wang, Y. Zhao, K. Tait, X. Liao, D. Schiferl, C. Zha, R.T. Downs, J. Qian, Y. Zhu, T. Shen. Proc. Natl. Acad. Sci. 101, 38, 13699 (2004).

[12] F. Zhang, J. Shen, J. Sun, D.G. McCartney. Carbon 44, 3136 (2006).

[13] I.O. Bashkin, V.I. Rashchupkin, A.F. Gurov, A.P. Moravsky, O.G. Rybchenkot, N.P. Kobelev. J. Phys.: Condens. Matter. 6, 7491 (1994).

[14] В.В. Бражкин, А.Г. Ляпин. УФН 166, 8, 893 (1996).

[15] P. Giannozzi, S. Baroni, N. Bonini, M. Calandra, R. Car, C. Cavazzoni, D. Ceresoli, G.L. Chiarotti, M. Cococcioni, I. Dabo, A.D. Corso, S. de Gironcoli, S. Fabris, G. Fratesi, R. Gebauer, U. Gerstmann, C. Gougoussis, A. Kokalj, M. Lazzeri, L. Martin-Samos, N. Marzari, F. Mauri, R. Mazzarello, S. Paolini, A. Pasquarello, L. Paulatto, C. Sbraccia1, S. Scandolo, G. Sclauzero, A.P. Seitsonen, A. Smogunov, P. Umari, R.M. Wentzcovitch. J. Phys.: Condens. Matter. 21, 39, 395502 (2009).

[16] J.P. Perdew, A. Zunger. Phys. Rev. B 23, 10, 5048 (1981).

[17] J.P. Perdew, K. Burke., M. Ernzerhof. Phys. Rev. Lett. 77, 18, 3865 (1996).

[18] H.J. Monkhorst, J.D. Pack. Phys. Rev. B 13, 12, 5188 (1976).

[19] Я.С. Уманский, Ю.А. Скаков, А.Н. Иванов, Л.Н. Расторгуев. Кристаллография, рентгенография и электронная микроскопия. Металлургия, М. (1982). 632 с.

[20] Е.А. Беленков, В.А. Грешняков. ФТТ 55, 8, 1640 (2013).

[21] J. Zhai, A. Wan, W. Wu. Mod. Phys. Lett. B 29, S1, 1530011 (2015).

[22] В.А. Грешняков, Е.А. Беленков. ЖТФ 86, 10, 20 (2016).

[23] В.А. Грешняков, Е.А. Беленков. ЖЭТФ 151, 2, 310 (2017).

Редактор Д.В. Жуманов 